\title{
GROUPS WITH FAITHFUL BLOCKS
}

\section{H. PAHLINGS}

ABSTRACT. A necessary and sufficient condition is given for a finite group to have a $p$-block with kernel $\{1\}$ 。 This extends a theorem of Gaschütz on the existence of a faithful irreducible representation of a finite group.

Let $G$ be a finite group and $F$ a field of characteristic $p \geq 0$. Then the group algebra $F G$ is a direct sum of two-sided ideals $B_{i}$ which are indecomposable as two-sided ideals $F G=B_{1} \oplus \cdots \oplus B_{n}$. The $B_{i}$ are called the block ideals of $F G$. If $e$ is a block idempotent, i.e. the identity element of a block ideal $B$, then a (left) $F G$-module $V$ is said to belong to the block $B \leftrightarrow e$ if $e V=V$.

Furthermore, if $p>0$ and $R$ is a complete discrete valuation ring with residue class field $F$ and quotient field $K$ of characteristic 0 , then every block idempotent $e$ can be lifted to a block idempotent $\check{e}$ of $R G$, which can be embedded in KG. A $K G$-module $V$ is also said to belong to the block $B \leftrightarrow e$ if $\check{e} V=V$.

For the case that $F$ and $K$ are splitting fields for $G, \mathrm{R}$. Brauer [1] has defined the kernel of the block $B \leftrightarrow e$ to be the intersection of the kernels of the (ordinary) irreducible $K$-representations of $G$ belonging to $B \leftrightarrow e$. Returning to the case where $F$ is an arbitrary field we feel that it is natural to make the following

Definition. The kernel $N(e)$ of a block $B \leftrightarrow e$ is the kernel of the $F$ representation of $G$, which is afforded by the block ideal $B$. Thus $N(e)=$ $\{g \in G \mid g e=e\}$.

We remark that this agrees with Brauer's definition as Proposition 1 (b) below shows, but differs slightly from the one used in [3] and [4], where the intersection $N^{*}(e)$ of the kernels of the irreducible $F$-representations of $G$ belonging to $B \leftrightarrow e$ was called the kernel of $B \leftrightarrow e$. The kernel $N(e)$ is uniquely determined by $N^{*}(e)$ and vice versa. In fact, by a result of

Received by the editors April 2, 1974.

AMS (MOS) subject classifications (1970). Primary 20C20, $20 \mathrm{C} 05$. sentations.

Key words and phrases. Group algebra, block, kernel of a block, faithful repre- 
Michler [3]

$$
N(e)=O_{p^{\prime}}\left(N^{*}(e)\right), \quad N^{*}(e) / N(e)=O_{p}(G / N(e))
$$

i.e. $N(e)$ is the maximal p-regular normal subgroup of $N^{*}(e)$ and $N^{*}(e) / N(e)$ is the maximal normal $p$-subgroup of $G / N(e)$. Here a normal subgroup is called $p$-regular, if its order is not divisible by $p$.

Proposition 1. (a) The kernel $N(e)$ of the block $B \leftrightarrow e$ is equal to the kernel of every principal indecomposable module belonging to $B \leftrightarrow e$.

(b) If $p>0$ and $R$ is a complete discrete valuation ring with residue class field $F$ and quotient field $K$ of characteristic 0 , then $N(e)$ is the intersection of the kernels of the irreducible $K$-representations belonging to $B \leftrightarrow e$.

Proof. (a) Obviously $N(e)$ is the intersection of the kernels of the principal indecomposable $F$-representations belonging to $B \leftrightarrow e$. Let $H$ be the kernel of a principal indecomposable module $F G u$ belonging to $B \leftrightarrow e$, where $u$ denotes a primitive idempotent of $F G$. $H$ must be $p$-regular, since if $u=\Sigma_{g \in G} \alpha_{g} g$, then $\alpha_{g}$ is constant on the cosets of $H$, because for $b \in H$, bu $=u$. Thus $u=\Sigma_{i=1}^{r} a_{i} g_{i}\left(\Sigma_{h \in H} b\right)$, where $g_{1}, \ldots g_{r}$ are coset representatives of $H$ in $G$, and

$$
u=u^{2}=|H| \sum_{i, j} a_{i} a_{j} g_{i} g_{j}\left(\sum_{h \in H} b\right),
$$

which would be 0 if $p$ divides $|H|$.

Hence one can form $s_{H}=|H|^{-1} \Sigma_{b \in H} b$ and one has $s_{H} u=u$. But $s_{H}$ is a central idempotent and $s_{H}=\Sigma_{N\left(e_{i}\right) \geq H} e_{i}$. Since $s_{H} u=u$, the block idempotent $e$ must occur in the sum, therefore $N(e) \geq H$.

(b) $K G \check{e}$ is the direct sum of those simple ideals, which have irreducible modules belonging to $B \leftrightarrow e$. Hence the intersection of the kernels of the irreducible $K$-representations belonging to $B \leftrightarrow e$ is $\{g \in G \mid g \check{e}=\check{e}\}$. Since $\check{e}$ is mapped onto $e$ under the natural map $R G \rightarrow F G$, one has obviously $\{g \in G \mid g \check{e}=\check{e}\} \leq N(e)$. Conversely, let $x \in N(e)$, hence $x e=e$. We denote the unique maximal ideal of $R$ by $\pi R$. If $x \check{e} \neq \check{e}$, there is a maximal number $k \geq 1$ such that $x \check{e}-\check{e} \in \pi^{k} R G$. Let $\check{e}=\Sigma_{g \in G} \alpha_{g} g\left(\alpha_{g} \in R\right)$; then $\alpha_{g} \equiv \alpha_{x g} \equiv \cdots \equiv \alpha_{x^{m-1}} \bmod \left(\pi^{k}\right)$, where $m$ is the order of $x$. Hence, if $g_{1}, \ldots, g_{r}$ are coset representatives of $\langle x\rangle$ in $G$,

$$
e=\sum_{i=1}^{r}\left(\alpha_{i}\left(1+x+\cdots+x^{m-1}\right)+b_{i}\right) g_{i}
$$

with $b_{i} \in \pi^{k} R G$ and $\alpha_{i}=\alpha_{g_{i}}$. But then 


$$
x \check{e}-\check{e}=(x \check{e}-\check{e}) \check{e}=\sum_{i=1}^{r}(x \check{e}-\check{e}) b_{i} g_{i} \in \pi^{2 k} R G,
$$

a contradiction.

The natural question-Which $p$-regular normal subgroups $H$ can be kernels of blocks?-can be reduced to the case $H=\{1\} . F G$ has a block with kernel $H$ if and only if $H$ is p-regular and $F(G / H)$ has a block with kernel \{1\}. This follows from the fact that if $H$ is $p$-regular and $s_{H}=|H|^{-1} \Sigma_{h \in H} h$, then $F G=F G s_{H} \oplus F G\left(1-s_{H}\right)$, where $F G s_{H} \cong F(G / H)$ is the direct sum of all block ideals of $F G$ with kernels containing $H$.

Proposition 2. FG bas a block with kernel $\{1\}$ if and only if the maximal p-regular normal subgroup $O_{p}(\operatorname{Soc}(G))$ of the socle of $G$ is generated by one class of conjugate elements of $G$.

For the case $p=0$ or $p$ a prime not dividing the order of $G$ this proposition contains the theorem of Gaschütz [2] (see also Žmud' [5]) on the existence of a faithful irreducible representation; for in this case the kernel of a block is simply the kernel of an irreducible representation, and $O_{p^{\prime}}(\operatorname{Soc}(G))=\operatorname{Soc}(G)$.

Proof of Proposition 2. It was shown in [4] that if $F G$ has a block with kernel $\{1\}$ then the same is true also for $F^{\prime} G$, where $F^{\prime}$ is any field with the same characteristic as $F$. Hence one can assume that $F$ is a splitting field for $G$.

Let $\Phi_{i}(1 \leq i \leq r)$ be the characters of the principal indecomposable representations of $F G$ and $\phi_{i}(1)$ be the degrees of the corresponding irreducible representations of $F G$. If $H$ is a $p$-regular normal subgroup of $G$, it follows from the orthogonality relations, for $G / H$ that

$$
\sum_{N\left(\Phi_{i}\right) \geq H} \phi_{i}(1) \Phi_{i}(x)= \begin{cases}|G / H| & \text { if } x \in H, \\ 0 & \text { if } x \notin H,\end{cases}
$$

where the sum ranges over all $i$ such that the kernel $N\left(\Phi_{i}\right)$ contains $H$.

If $M_{1}, \ldots, M_{m}$ are the minimal normal $p$-regular subgroups of $G$, then

$$
\begin{aligned}
\psi(x) & =\sum_{N\left(\Phi_{i}\right)=\{1\}} \phi_{i}(1) \Phi_{i}(x) \\
& =\sum_{i=1}^{r} \phi_{i}(1) \Phi_{i}(x)+\sum_{k=1}^{m}(-1)^{k} \sum_{1 \leq j} \sum_{1} \sum_{N\left(\boldsymbol{S}_{k} \leq m\right.} \sum_{i} M_{j} \ldots M_{j}{ }_{j}(1) \Phi_{i}(x),
\end{aligned}
$$


where an empty sum is understood to be 0 . Hence

$$
\psi(1)=|G|+\sum_{k=1}^{m}(-1)^{k} \sum_{1 \leq j_{1}<\cdots<j_{k} \leq m}\left|G: M_{j_{1}} \cdots M_{j_{k}}\right| \cdot
$$

The lattice of normal subgroups of $G$, which are contained in $Q=$ $O_{p}{ }^{\prime}(\operatorname{Soc}(G))$ has a duality $\epsilon$ such that $(M N)^{\epsilon}=M^{\epsilon} \cap N^{\epsilon},(M \cap N)^{\epsilon}=M^{\epsilon} N^{\epsilon}$. and $\left|N^{\epsilon}\right|=|Q: N|$. If $N_{1}, \ldots, N_{m}$ are the normal subgroups of $G$ which are maximal in $Q$, then

$$
\psi(1)=|G|+\sum_{k=1}^{m}(-1)^{k} \sum_{1 \leq j_{1}<\cdots<j_{k} \leq m}\left|G: N_{j_{1}}^{\epsilon} \cdots N_{j_{k}}^{\epsilon}\right| \cdot
$$

Since $\left|Q: N_{j_{1}}^{\epsilon} \cdots N_{j_{k}}^{\epsilon}\right|=\left|N_{j_{1}} \cap \cdots \cap N_{j_{k}}\right|$, it follows that

$$
\psi(1)=|G: Q||Q| \bigcup_{j=1}^{m} N_{j} \mid
$$

and this is different from zero if and only if $Q$ is generated by one class of conjugate elements of G. Q.E.D.

\section{REFERENCES}

1. R. Brauer, Some applications of the theory of blocks of characters of finite groups. I, J. Algebra 1 (1964), 152-167. MR 29 \#5920.

2. W. Gaschütz, Endliche Gruppen mit treuen absolut-irreduziblen Darstellungen, Math. Nachr. 12 (1954), 253-255. MR 16, 671.

3. G. O. Michler, The kernel of a block of a group algebra, Proc. Amer. Math. Soc. 37 (1973), 47-49.: MR 46 \#9151.

4. H. Pahlings, Über die Kerne von Blöcken einer Gruppenalgebra, Arch. Math. 25 (1974), 121-124.

5. E. M. Žmud', On the kernels of homomorphisms of linear representations of a finite group, Mat. Sb. 44(86) (1958), 353-408. (Russian) MR 20 \#5236.

DEPARTMENT OF MATHEMATICS, UNIVERSITY OF GIESSEN, 63 GIESSEN, FEDERAL REPUBLIC OF GERMANY 